On the gravitoelectromagnetic stress-energy tensor

This article has been downloaded from IOPscience. Please scroll down to see the full text article.

1999 Class. Quantum Grav. 161137

(http://iopscience.iop.org/0264-9381/16/4/004)

View the table of contents for this issue, or go to the journal homepage for more

Download details:

IP Address: 128.206.162.204

The article was downloaded on 28/09/2010 at 19:46

Please note that terms and conditions apply. 


\title{
On the gravitoelectromagnetic stress-energy tensor
}

\author{
Bahram Mashhoon†, James C McClune† and Hernando Quevedo \\ $\dagger$ Department of Physics and Astronomy, University of Missouri-Columbia, Columbia, MO \\ 65211, USA \\ \$Instituto de Ciencias Nucleares, Universidad Nacional Autónoma de México, AP 70-543, \\ 04510 México DF, Mexico
}

Received 14 May 1998, in final form 21 December 1998

\begin{abstract}
We study the pseudo-local gravitoelectromagnetic stress-energy tensor for an arbitrary gravitational field within the framework of general relativity. It is shown that there exists a current of gravitational energy around a rotating mass. This gravitational analogue of the Poynting flux is evaluated for certain classes of observers in the Kerr field.
\end{abstract}

PACS numbers: 0420C, 0420M, 0470

\section{Introduction}

Imagine a packet of long-wavelength gravitational waves incident on a Keplerian binary system. In the lowest (quadrupole) approximation, the waves exchange energy and angular momentum with the self-gravitating system. To describe this interaction in detail, it is evident that an essentially local measure of the energy and stress carried by the waves is necessary. Therefore, there must be a somewhat local way to describe the energy and momentum stored in the gravitational field. A partial (i.e. gravitoelectromagnetic) solution of this general problem within the framework of general relativity is essentially contained in a recent paper [1]. The present work is concerned with the extension of our approach and some of its general consequences. Our paper on the gravitational superenergy tensor [1] should be consulted for much of the background material; however, we have attempted to make the present paper essentially self-contained.

The standard (Landau-Lifshitz) gravitational stress-energy pseudotensor is useful in general relativity only in a global sense for asymptotically flat spacetimes. For many applications, however, a local measure of the stress-energy content of an arbitrary gravitational field would be helpful. It is possible to provide such a measure - though only as an approximation-for the gravitoelectromagnetic part of the field as shown in our recent paper [1]. As expected, the gravitoelectromagnetic stress-energy tensor has general properties that are rather similar to those of the Maxwell stress-energy tensor in electrodynamics. For a Ricciflat spacetime, this approach provides an average measure of the gravitational stress-energy content that is proportional to the Bel-Robinson tensor. Thus our derivation of the gravitational stress-energy tensor provides a natural physical interpretation of the Bel-Robinson tensor that has been used frequently in numerical relativity [2].

The curved spacetimes of general relativity are not asymptotically flat in general; therefore, the usual concepts of energy, momentum and stress do not make sense in the standard interpretation of general relativity. Nevertheless, it is possible to introduce a pseudo-local 
gravitoelectromagnetic ('GEM') stress-energy tensor via a certain averaging procedure. This approach is discussed briefly in the next section and the general properties of the GEM tensor are described. In sections 3 and 4, special gravitational fields are considered - such as the charged Kerr-Taub-NUT spacetime- and their GEM stress-energy tensors are evaluated. Moreover, the existence of a steady current of gravitational field energy around a rotating mass is predicted and some of its properties are briefly studied in section 5 . We employ units such that the speed of light $c=1$; furthermore, the spacetime metric has signature +2 , Greek indices run from $0-3$ and Latin indices run from 1-3.

\section{GEM stress-energy tensor}

Consider a free test observer in a gravitational field following a worldline $\mathcal{C}$ and let $\tau$ be the proper time along $\mathcal{C}$. In a Fermi coordinate system $X^{\alpha}=(\tau, \boldsymbol{X})$ along the geodesic path of the observer, the physical content of the spacetime interval can be described in terms of a gravitoelectric potential $\Phi$, a gravitomagnetic vector potential $\boldsymbol{A}$ and a spatial tensor potential $S$. In fact, the metric in the Fermi frame is given by ${ }^{F} g_{\mu \nu}=\eta_{\mu \nu}+{ }^{F} h_{\mu \nu}$, where ${ }^{F} h_{00}=2 \Phi$, ${ }^{F} h_{0 i}=-2 A_{i}$ and ${ }^{F} h_{i j}=S_{i j}$. To the lowest order in spatial Fermi coordinates away from $\mathcal{C}$,

$$
\begin{aligned}
& \Phi=-\frac{1}{2}{ }^{F} R_{0 i 0 j}(\tau) X^{i} X^{j}, \\
& A_{i}=\frac{1}{3}{ }^{F} R_{0 j i k}(\tau) X^{j} X^{k}, \\
& S_{i j}=-\frac{1}{3}{ }^{F} R_{i k j l}(\tau) X^{k} X^{l},
\end{aligned}
$$

where ${ }^{F} R_{\alpha \beta \gamma \delta}=R_{\mu \nu \rho \sigma} \lambda_{(\alpha)}^{\mu} \lambda_{(\beta)}^{v} \lambda_{(\gamma)}^{\rho} \lambda_{(\delta)}^{\sigma}$ is the spacetime curvature measured by the observer, i.e. it is the projection of the Riemann curvature tensor along the nonrotating tetrad system $\lambda^{\mu}{ }_{(\alpha)}(\tau)$ carried by the observer at $C^{\mu}:(\tau, \mathbf{0})$ in the Fermi system. In general, the locally measured components of the Riemann curvature tensor, $R_{\mu \nu \rho \sigma} \lambda_{(\alpha)}^{\mu} \lambda_{(\beta)}^{v} \lambda_{(\gamma)}^{\rho} \lambda_{(\delta)}^{\sigma}$, can be represented as a symmetric $6 \times 6$ matrix $\mathcal{R}=\left(\mathcal{R}_{I J}\right)$, where the indices $I$ and $J$ range over the set $(01,02,03,23,31,12)$. The symmetries of the Riemann tensor make it possible to write

$$
\mathcal{R}=\left[\begin{array}{cc}
E & B \\
B^{T} & D
\end{array}\right]
$$

where $E$ and $D$ are symmetric $3 \times 3$ matrices and $B$ is traceless. Here $E$ and $B$ represent the 'electric' and 'magnetic' components of the spacetime curvature, respectively, and $D$ represents the 'spatial' components. The curvature of the gravitational field is thus determined by the three matrices $E, B$ and $D$. In a Ricci-flat spacetime, $R_{\mu \nu}=0, D=-E$ so that the spatial components of the curvature are completely determined by the electric components; moreover, $E$ is traceless and $B$ is symmetric $\left(B^{T}=B\right)$.

Using the potentials $\Phi$ and $A$, it is natural to define the gravitoelectric and gravitomagnetic fields in complete analogy with electrodynamics. Keeping only the lowest-order terms in the spatial Fermi coordinates, we find

$$
\begin{aligned}
\mathcal{E}_{i} & ={ }^{F} R_{0 i 0 j}(\tau) X^{j}, \\
\mathcal{B}_{i} & =-\frac{1}{2} \epsilon_{i j k}{ }^{F} R_{j k 0 l}(\tau) X^{l} .
\end{aligned}
$$

It turns out that these fields can be combined in a gravitoelectromagnetic 'Faraday' tensor given by

$$
\mathcal{F}_{\alpha \beta}=-{ }^{F} R_{\alpha \beta 0 l}(\tau) X^{l}
$$


It is then natural to construct from the GEM field tensor (7) the corresponding 'Maxwell' stress-energy tensor

$$
\mathcal{T}^{\alpha \beta}=\frac{1}{4 \pi}\left(\mathcal{F}_{\gamma}^{\alpha} \mathcal{F}^{\beta \gamma}-\frac{1}{4} \eta^{\alpha \beta} \mathcal{F}_{\gamma \delta} \mathcal{F}^{\gamma \delta}\right)
$$

which is symmetric and traceless by construction just as in electromagnetism. We can also define the GEM angular momentum density tensor

$$
\mathcal{J}^{\alpha \beta \gamma}=\left(X^{\alpha}-C^{\alpha}\right) \mathcal{T}^{\beta \gamma}-\left(X^{\beta}-C^{\beta}\right) \mathcal{T}^{\alpha \gamma},
$$

where $C^{\alpha}:(\tau, \mathbf{0})$ denote the coordinates of the fiducial test observer at the centre of the Fermi system (i.e. the spatial origin of Fermi coordinates). Thus $\mathcal{J}^{\alpha \beta \gamma}$ vanishes at the spacetime position of the test observer by construction. The GEM angular momentum tensor is then defined by

$$
J^{\alpha \beta}=\int \mathcal{J}^{\alpha \beta \gamma} \mathrm{d}^{3} \Sigma_{\gamma}
$$

as in standard field theory, except that the relevant three-dimensional (3D) volume must be within the Fermi system and consistent with the approximation scheme under consideration here.

The stress-energy tensor (8) has the property that it vanishes along the worldline $\mathcal{C}$ by Einstein's principle of equivalence; however, it is in general nonzero on geodesic paths in the neighbourhood of the fiducial worldline. Measurement of such field quantities does not occur at a point in space; in fact, an averaging process is indispensable. An interesting and useful measure of the gravitoelectromagnetic stress-energy in the immediate vicinity of the observer may be obtained by averaging the stress-energy tensor over a sphere of radius $\epsilon L$ at each instant of proper time along the fiducial path. Here $\epsilon, 0<\epsilon \ll 1$, is a small parameter and $L$ is an intrinsic length-scale characteristic of the gravitational field. The form of the tensor which is averaged in the Fermi frame is such that the same result is in effect obtained whether one averages over the surface or the volume of an infinitesimal sphere about the spatial origin of Fermi coordinates, since the difference could be absorbed in the definition of $L$. The resulting average stress-energy tensor is then physically defined only up to an overall positive constant scale factor.

It follows from these considerations that the average gravitoelectromagnetic stress-energy content of the field as determined by the observer at the event under consideration is given by the projection of a symmetric traceless stress-energy tensor $\tilde{T}_{\mu \nu}$ on the observer's frame, i.e.

$$
\tilde{T}_{(\alpha)(\beta)}=\frac{L^{2}}{G_{0}} \tilde{T}_{\mu \nu \rho \sigma} \lambda_{(\alpha)}^{\mu} \lambda_{(\beta)}^{\nu} \lambda_{(0)}^{\rho} \lambda_{(0)}^{\sigma},
$$

where $L$ is the constant invariant length characteristic of the gravitational field, $G_{0}$ is Newton's constant and $\tilde{T}_{\mu \nu \rho \sigma}$ is given by

$$
\tilde{T}_{\mu \nu \rho \sigma}=\frac{1}{2}\left(R_{\mu \xi \rho \zeta} R_{\nu \sigma}^{\xi \xi}+R_{\mu \xi \sigma \zeta} R_{\nu \rho}^{\xi \zeta}\right)-\frac{1}{4} g_{\mu \nu} R_{\alpha \beta \rho \gamma} R_{\sigma}^{\alpha \beta \gamma} .
$$

This tensor is symmetric and traceless in its first pair of indices and symmetric in its second pair of indices and reduces to the completely symmetric and traceless Bel-Robinson tensor, $T_{\mu \nu \rho \sigma}$, for a Ricci-flat spacetime (cf appendix A). Then $\tilde{T}_{(\alpha)(\beta)}$ reduces in turn to $T_{(\alpha)(\beta)}$, which we call the 'gravitational stress-energy tensor' since the curvature is completely characterized in this case by its GEM part.

Equations (11) and (12) originate from local dynamical considerations [1]. In a Fermi frame established along the worldline of a geodesic observer, the measured gravitoelectric and gravitomagnetic fields to linear order in the spatial distance away from the fiducial path can be combined to form a gravitoelectromagnetic Faraday tensor and the corresponding GEM 
Maxwell stresses and angular momentum densities. If equation (9) is integrated over the volume of the infinitesimal sphere, i.e. if in equation (10) we integrate at a given $\tau$ over the sphere of centre $C^{\mu}:(\tau, \mathbf{0})$ and radius $\epsilon L$, then we find that $J^{\alpha \beta}=0$. This means that the local centre of mass of the sphere is indeed $C^{\mu}:(\tau, \mathbf{0})$ as expected; moreover, the field angular momentum $J^{i j}$ contained in this sphere vanishes at the linear order of approximation for the fields (5) and (6) under consideration here.

We find that, in general,

$$
\begin{aligned}
& \tilde{T}_{(0)(0)}=\frac{L^{2}}{2 G_{0}} \operatorname{tr}\left(E^{2}+B^{T} B\right), \\
& \tilde{T}_{(0)(i)}=\frac{L^{2}}{G_{0}} \epsilon_{i j k}(E B)_{j k}, \\
& \tilde{T}_{(i)(j)}=\frac{L^{2}}{2 G_{0}}\left[-2\left(E^{2}+B^{T} B\right)_{i j}+\delta_{i j} \operatorname{tr}\left(E^{2}+B^{T} B\right)\right],
\end{aligned}
$$

so that $\tilde{T}_{(\alpha)(\beta)}$ only contains the 'electric' and 'magnetic' parts of the spacetime curvature and not the 'spatial' part. It is immediately clear from equation (13) that $\tilde{T}_{(0)(0)}$ is positive definite; hence $\tilde{T}_{(0)(0)}=0$ implies that $E=0$ and $B=0$ and the spacetime curvature is thus purely 'spatial' for the observer in this case. Moreover, by writing the matrices $E$ and $B$ in equations (13)-(15) in component form and repeated application of the simple relations $|\eta+\nu| \leqslant|\eta|+|\nu|$ and $|\eta \nu| \leqslant \frac{1}{2}\left(\eta^{2}+\nu^{2}\right)$ for arbitrary real numbers $\eta$ and $\nu$, it is straightforward to show that

$$
\left|\tilde{T}_{(0)(i)}\right| \leqslant \tilde{T}_{(0)(0)}
$$

and

$$
\left|\tilde{T}_{(i)(j)}\right| \leqslant \tilde{T}_{(0)(0)} .
$$

Thus the gravitational Poynting vector is always timelike or null and the absolute magnitude of a gravitoelectromagnetic stress is always bounded above by the local average density. These relations are reminiscent of Maxwell's electrodynamics.

It is interesting to note that, in general,

$$
\frac{1}{4} R_{\mu \nu \rho \sigma} R^{\mu \nu \rho \sigma}=\operatorname{tr}\left(E^{2}-2 B^{T} B+D^{2}\right),
$$

and

$$
\frac{1}{16} R_{\alpha \beta}{ }^{\mu v} R^{\alpha \beta \rho \sigma} e_{\mu v \rho \sigma}=\operatorname{tr}(E B-B D),
$$

where $e_{\mu \nu \rho \sigma}$ is the alternating tensor $e_{\mu \nu \rho \sigma}=(-g)^{1 / 2} \epsilon_{\mu \nu \rho \sigma}$ with $\epsilon_{0123} \equiv 1$.

It is possible, in principle, to study the GEM stress-energy tensor for the known exact solutions of the gravitational field equations; in particular, astrophysically relevant solutions for stars, collapsing configurations, radiative spacetimes and cosmological models should be investigated. Only a beginning is made in this paper; a full discussion is beyond the scope of the present work.

Let us now assume that $R_{\mu \nu}=0$. In this case, $D=-E, E$ is traceless and $B$ is symmetric. Thus $E$ and $B$ characterize the whole gravitational field in this case. This is illustrated in the next section via approximate gravitational field solutions that are Ricci flat. Moreover, in the Ricci-flat case, as noted already by Matte [4], the curvature invariants (18) and (19) divided by 2 take the forms $\operatorname{tr}\left(E^{2}-B^{2}\right)$ and $\operatorname{tr}(E B)$, respectively, that are familiar from electrodynamics. 
The gravitoelectromagnetic stress-energy tensor $\tilde{T}_{\mu \nu}$ reduces in the Ricci-flat case to the gravitational stress-energy tensor $T_{\mu \nu}$ discussed previously [1]. The Riemann tensor reduces to the Weyl tensor in the latter case; therefore, the connection between $\tilde{T}_{\mu \nu}$ and $T_{\mu \nu}$ can be worked out in general using

$R_{\mu \nu \rho \sigma}=C_{\mu \nu \rho \sigma}+\frac{1}{2}\left(R_{\mu \rho} g_{\nu \sigma}+R_{\nu \sigma} g_{\mu \rho}-R_{\mu \sigma} g_{\nu \rho}-R_{\nu \rho} g_{\mu \sigma}\right)-\frac{1}{6} R\left(g_{\mu \rho} g_{\nu \sigma}-g_{\mu \sigma} g_{\nu \rho}\right)$,

where $R_{\mu \nu}$ and $R$ are given in terms of the stress-energy tensor of the source of the field via the gravitational field equations. It is more interesting, however, to work out $T_{\mu \nu}$ explicitly for certain approximate solutions of the gravitational field equations in which nonlinearities are neglected for the sake of simplicity. This is done in the next section.

\section{Linear gravitational fields}

Let us first imagine linear gravitational waves given by $g_{\mu \nu}=\eta_{\mu \nu}+h_{\mu \nu}$, where a gauge is chosen such that $h_{0 \mu}=0, h^{i j}{ }_{j}=0$ and $\operatorname{tr}\left(h_{i j}\right)=0$. All static observers follow geodesics of this gravitational field. The gravitational wave amplitudes $h_{i j}(t, x)$ satisfy the wave equation; therefore, we consider for the sake of simplicity a plane monochromatic wave of frequency $\omega_{g}$ propagating along the $x$-direction. Then, the gravitoelectric and gravitomagnetic fields, as measured by geodesic observers at fixed spatial positions, can be expressed in this case as

$E=\frac{1}{2} \omega_{g}^{2}\left[\begin{array}{ccc}0 & 0 & 0 \\ 0 & h_{+} & h_{\times} \\ 0 & h_{\times} & -h_{+}\end{array}\right], \quad B=\frac{1}{2} \omega_{g}^{2}\left[\begin{array}{ccc}0 & 0 & 0 \\ 0 & h_{\times} & -h_{+} \\ 0 & -h_{+} & -h_{\times}\end{array}\right]$,

where $2 E_{i j}=\omega_{g}^{2} h_{i j}$, so that $h_{+}$and $h_{\times}$represent the two independent linear polarization states of the wave. It is clear that a wavepacket may be formed by a simple superposition of the fields given by equation (21). It follows from equations (13)-(15) that for each monochromatic component

$$
\left(T^{(\alpha)(\beta)}\right)=\frac{L^{2} \omega_{g}^{4}}{2 G_{0}}\left(h_{+}^{2}+h_{\times}^{2}\right)\left[\begin{array}{cccc}
1 & 1 & 0 & 0 \\
1 & 1 & 0 & 0 \\
0 & 0 & 0 & 0 \\
0 & 0 & 0 & 0
\end{array}\right],
$$

which should be compared and contrasted with the corresponding result obtained using the Landau-Lifshitz pseudotensor $t_{\mu \nu}^{L-L}$ (cf the appendix of [5]). In fact, $t_{\mu \nu}^{L-L}$ is in general gauge dependent and its trace,

$$
\operatorname{tr}\left(t_{\mu \nu}^{L-L}\right)=\frac{1}{32 \pi G_{0}} h_{\alpha \beta, \gamma} h^{\alpha \beta, \gamma},
$$

is nonzero [5]; that is, equation (23) is nonzero for a general wavepacket, but vanishes for a plane wave. On the other hand, the local gravitational stress-energy tensor is gauge invariant and traceless just as in Maxwell's electrodynamics.

Let us next imagine the post-Newtonian field of a system with mass $M$ and angular momentum $J$ given in the linear approximation by the standard metric

$-\mathrm{d} s^{2}=-\left(1-2 \frac{G_{0} M}{|\boldsymbol{x}|}\right) \mathrm{d} t^{2}-4 \frac{G_{0} \mathrm{~d} t}{|\boldsymbol{x}|^{3}} \epsilon_{i j k} J^{i} x^{j} \mathrm{~d} x^{k}+\left(1+2 \frac{G_{0} M}{|\boldsymbol{x}|}\right) \delta_{i j} \mathrm{~d} x^{i} \mathrm{~d} x^{j}$.

An observer that is initially at rest in this field will not remain at a constant position $\boldsymbol{x}$; however, this motion can be neglected in the linear order of approximation under discussion 
in this section. It follows that [6]

$$
\begin{aligned}
& E_{i j}=\frac{G_{0} M}{|\boldsymbol{x}|^{3}}\left(\delta_{i j}-3 \hat{x}^{i} \hat{x}^{j}\right), \\
& B_{i j}=-3 \frac{G_{0} J}{|\boldsymbol{x}|^{4}}\left[\hat{x}^{i} \hat{\boldsymbol{J}}^{j}+\hat{x}^{j} \hat{\boldsymbol{J}}^{i}+\left(\delta_{i j}-5 \hat{x}^{i} \hat{x}^{j}\right) \hat{\boldsymbol{x}} \cdot \hat{\boldsymbol{J}}\right],
\end{aligned}
$$

where $\hat{\boldsymbol{x}}=\boldsymbol{x} /|\boldsymbol{x}|$ is the unit position vector of the static observer under consideration here. We find that

$$
\begin{aligned}
T^{(0)(0)}= & \frac{3 G_{0} L^{2}}{|\boldsymbol{x}|^{6}}\left\{M^{2}+3 \frac{J^{2}}{|\boldsymbol{x}|^{2}}\left[1+2(\hat{\boldsymbol{x}} \cdot \hat{\boldsymbol{J}})^{2}\right]\right\}, \\
T^{(0)(i)}= & \frac{9 G_{0} L^{2} M J}{|\boldsymbol{x}|^{7}}(\hat{\boldsymbol{J}} \times \hat{\boldsymbol{x}})^{i}, \\
T^{(i)(j)}= & \frac{3 G_{0} L^{2} M^{2}}{|\boldsymbol{x}|^{6}}\left(\frac{2}{3} \delta_{i j}-\hat{x}^{i} \hat{x}^{j}\right)+\frac{9 G_{0} L^{2} J^{2}}{|\boldsymbol{x}|^{8}}\left[\delta_{i j}-\hat{x}^{i} \hat{x}^{j}-\hat{\boldsymbol{J}}^{i} \hat{\boldsymbol{J}}^{j}\right. \\
& \left.+2(\hat{\boldsymbol{x}} \cdot \hat{\boldsymbol{J}})\left(\hat{x}^{i} \hat{\boldsymbol{J}}^{j}+\hat{x}^{j} \hat{\boldsymbol{J}}^{i}\right)+(\hat{\boldsymbol{x}} \cdot \hat{\boldsymbol{J}})^{2}\left(\delta_{i j}-5 \hat{x}^{i} \hat{x}^{j}\right)\right] .
\end{aligned}
$$

The gravitational Poynting vector (28) is in this case analogous to the familiar circumstance in electrodynamics involving the exterior field of a static nearly spherical system with a net electric charge and a constant magnetic dipole moment. The analogous gravitational properties would be mass $M$ and spin $J$, respectively. The Poynting vector, which is the cross product of a radial electric field and a dipolar magnetic field, indicates a steady energy flux around the source just as equation (28) indicates a steady gravitational energy current around a mass flowing in the same sense as the rotation of the body. Let us briefly digress here and mention that in the electromagnetic case, the Poynting energy flux produces a gravitoelectromagnetic field even when the material source itself does not rotate [7]. That is, the gravitational field is caused by the total stress-energy tensor, which in this case would originate from the static electromagnetic source together with the electromagnetic field that involves the steady energy flux.

The local average stress-energy tensor (27)-(29) should be useful in the post-Newtonian investigation of the dynamics of particles in the exterior gravitational field of a rotating mass.

\section{Kerr field}

To investigate further the nature of the steady gravitational energy flux around a rotating mass, it proves interesting to study the GEM stress-energy tensor for the Kerr field. Indeed, the study of the generalized Kerr spacetime is important due to its possible physical significance in connection with the complete gravitational collapse of matter [8]; however, we limit our treatment here to the charged Kerr-Taub-NUT spacetime for the sake of simplicity. To this end, let us imagine a set of observers following geodesic paths with nonrotating tetrads along their paths. Suppose that at some initial instant of time, the tetrad frames coincide with the natural tetrad system $\lambda^{\mu}{ }_{(\alpha)}$ of the charged Kerr-Taub-NUT spacetime. We are interested in the measured GEM stress-energy tensor at this initial time. It turns out that in terms of Schwarzschild-like coordinates $(t, r, \theta, \phi), \omega^{(\alpha)}=\lambda_{\mu}^{(\alpha)} \mathrm{d} x^{\mu}$ are given by

$$
\begin{aligned}
\omega^{(0)} & =\left(\frac{\Delta}{\Sigma}\right)^{1 / 2}\left[\mathrm{~d} t-\left(a \sin ^{2} \theta-2 l \cos \theta\right) \mathrm{d} \phi\right], \\
\omega^{(1)} & =\left(\frac{\Sigma}{\Delta}\right)^{1 / 2} \mathrm{~d} r,
\end{aligned}
$$




$$
\begin{aligned}
& \omega^{(2)}=\Sigma^{1 / 2} \mathrm{~d} \theta, \\
& \omega^{(3)}=\Sigma^{-1 / 2}\left[\left(r^{2}+a^{2}+l^{2}\right) \mathrm{d} \phi-a \mathrm{~d} t\right] \sin \theta, \\
& \Delta=r^{2}+a^{2}-l^{2}-2 m r+q^{2}, \\
& \Sigma=r^{2}+(a \cos \theta+l)^{2} .
\end{aligned}
$$

Here $m, a, l$ and $q$ are, respectively, the mass parameter, angular momentum per unit mass, Taub-NUT parameter and the charge parameter of the source. Only positive square roots are intended throughout this paper.

The components of the GEM stress-energy tensor in this tetrad system can be determined from the components of the curvature tensor. It is convenient to express the latter in the $S O(3, C)$ representation given by $M+P-\mathrm{i}(N+Q)$, where $M, N, P$ and $Q$ are $3 \times 3$ matrices in the bivector representation of the curvature tensor (4):

$$
\mathcal{R}=\left[\begin{array}{cc}
M & N \\
N & -M
\end{array}\right]+\left[\begin{array}{cc}
P & Q \\
Q & -P
\end{array}\right] .
$$

For the tetrad given above, we find [9]

$$
\begin{aligned}
& M-\mathrm{i} N=\left[\frac{m+\mathrm{i} l}{(r+\mathrm{i} \chi)^{3}}-\frac{q^{2}}{\Sigma(r+\mathrm{i} \chi)^{2}}\right] \operatorname{diag}(-2,1,1), \\
& P-\mathrm{i} Q=\frac{q^{2}}{\Sigma^{2}} \operatorname{diag}(1,0,0),
\end{aligned}
$$

where $\chi=a \cos \theta+l$. The resulting GEM stress-energy tensor is then given by

$$
\begin{aligned}
\frac{G_{0}}{L^{2}}\left(\tilde{T}^{(\mu)(\nu)}\right)= & \frac{m^{2}+l^{2}}{\Sigma^{3}} \operatorname{diag}(3,-1,2,2)+\frac{q^{2}}{\Sigma^{4}} \operatorname{diag}(2 \alpha, 2 \beta, \alpha-\beta, \alpha-\beta) \\
& +\frac{\gamma q^{2}}{\Sigma^{5}} \operatorname{diag}(1,-1,1,1),
\end{aligned}
$$

where

$$
\begin{aligned}
& \alpha=\frac{3}{4} q^{2}-2 l \chi, \\
& \beta=\frac{1}{4} q^{2}-2 m r, \\
& \gamma=4 r^{2}\left(q^{2}-2 l \chi-2 m r\right) .
\end{aligned}
$$

The diagonal form of the stress-energy tensor turns out to be consistent with our approximate treatment in section 3; in fact, this point will be discussed further in section 5.

Let us next consider the pure Kerr geometry $(l=0, q=0)$ and evaluate the gravitational stress-energy tensor for specific test observers. To this end, we first consider a free observer moving along the axis of axial symmetry such that far from the source $(r \rightarrow \infty)$ the observer has speed $\beta_{0}$. The equations of motion of the observer are given by

$$
\begin{aligned}
& \frac{\mathrm{d} t}{\mathrm{~d} \tau}=\gamma_{0} \frac{\Lambda^{2}}{\Delta}, \\
& \frac{\mathrm{d} r}{\mathrm{~d} \tau}= \pm\left(\gamma_{0}^{2}-1+\frac{2 m r}{\Lambda^{2}}\right)^{1 / 2},
\end{aligned}
$$

where $\gamma_{0}$ is the Lorentz factor at infinity $\gamma_{0}=\left(1-\beta_{0}^{2}\right)^{-1 / 2}$, and

$$
\Lambda=\left(r^{2}+a^{2}\right)^{1 / 2} \text {. }
$$


The spatial triad is chosen so that $\lambda_{(1)}^{\mu}$ is along the radial direction (i.e. the $z$-axis) and is given by

$$
\lambda_{(1)}^{\mu}=\left[\dot{r} \frac{\Lambda^{2}}{\Delta}, \gamma_{0}, 0,0\right],
$$

where $\dot{r}=\mathrm{d} r / \mathrm{d} \tau$. Due to rotational symmetry about the direction of motion, there is a simple degeneracy in the choice of $\lambda_{(2)}^{\mu}$ and $\lambda_{(3)}^{\mu}$, which are independent directions on a sphere of radius $\Lambda$. It suffices that $\lambda_{(\alpha)}^{\mu}$ should be an orthonormal tetrad system, then the curvature as measured by the observer is given by

$$
E=\frac{m r\left(r^{2}-3 a^{2}\right)}{\Lambda^{6}} \operatorname{diag}(-2,1,1), \quad B=\frac{m a\left(3 r^{2}-a^{2}\right)}{\Lambda^{6}} \operatorname{diag}(-2,1,1),
$$

so that the gravitoelectric and the gravitomagnetic parts of the curvature are parallel. The gravitational stress-energy tensor in this case turns out to be

$$
\left(T_{(\alpha)(\beta)}\right)=\frac{L^{2} m^{2}}{G_{0} \Lambda^{6}} \operatorname{diag}(3,-1,2,2)
$$

just as in (39) along the axis of symmetry for $l=0$ and $q=0$. This diagonal tensor is always regular since the observer can simply pass through the ring singularity.

It is important to recognize that equations (47) and (48) do not depend on $\beta_{0}$ at all. This important circumstance is a consequence of the fact that the axis of symmetry provides two special tidal directions of the Kerr field so that the curvature is independent of any Lorentz boosts along the ingoing and outgoing directions [6]. It follows that $T_{(\alpha)(\beta)}$ would then be independent of $\beta_{0}$ as well. This situation has a direct analogue in electrodynamics; that is, for an electromagnetic field that is not null one can always find a Lorentz frame in which the electric and magnetic fields are parallel. Any boost along the common direction of the fields leaves the fields as well as the corresponding electromagnetic stress-energy tensor invariant. It follows that (48) is an example of a general result: there exist special tidal directions at each event in a vacuum spacetime of type $D$ in the Petrov classification such that the curvature as well as the GEM stress-energy tensor remain invariant under boosts along these directions $[10,11]$.

It is interesting to consider a free test observer falling from rest at infinity in the equatorial plane of a Kerr system $\left(\beta_{0}=0, \theta=\pi / 2\right)$ with zero orbital angular momentum ('radial motion'). The geodesic path of the observer is given in $(t, r, \theta, \phi)$ coordinates by

$$
\begin{aligned}
& \dot{t}=\frac{1}{\Delta}\left(\Lambda^{2}+\frac{2 m a^{2}}{r}\right), \\
& \dot{r}^{2}=\frac{2 m \Lambda^{2}}{r^{3}}, \\
& \dot{\phi}=\frac{2 m a}{r \Delta} .
\end{aligned}
$$

Both incoming as well as outgoing geodesics are described by these equations. We consider a nonrotating spatial triad along the path given by

$$
\begin{aligned}
& \lambda_{(1)}^{\mu}=\left[\frac{\Lambda}{\Delta} r \dot{r}, \frac{\Lambda}{r}, 0, \frac{a r \dot{r}}{\Delta \Lambda}\right], \\
& \lambda_{(2)}^{\mu}=\left[0,0, \frac{1}{r}, 0\right], \\
& \lambda_{(3)}^{\mu}=\left[-\frac{2 m a \Lambda}{r \Delta},-\frac{a \dot{r}}{\Lambda}, 0, \frac{\Lambda}{\Delta}\left(1-\frac{2 m}{r}\right)\right],
\end{aligned}
$$


such that as $r \rightarrow \infty$ they correspond to the spherical coordinate axes [12]. The Riemann tensor as measured by the (ingoing or outgoing) observer is given by

$E=\frac{m}{r^{3}} \operatorname{diag}(-2,1,1)+\frac{3 m a^{2}}{r^{5}} \operatorname{diag}(-1,1,0), \quad B=\frac{3 m a \Lambda}{r^{5}}\left[\begin{array}{lll}0 & 1 & 0 \\ 1 & 0 & 0 \\ 0 & 0 & 0\end{array}\right]$.

Similarly, the nonzero components of the gravitational stress-energy tensor are independent of the direction of motion of the observer and are given by

$$
\begin{aligned}
& T^{(0)(0)}=\frac{3 L^{2} m^{2}}{G_{0} r^{10}}\left(r^{4}+6 r^{2} a^{2}+6 a^{4}\right), \\
& T^{(1)(1)}=-\frac{L^{2} m^{2}}{G_{0} r^{8}}\left(r^{2}+3 a^{2}\right), \\
& T^{(2)(2)}=\frac{L^{2} m^{2}}{G_{0} r^{8}}\left(2 r^{2}+3 a^{2}\right), \\
& T^{(3)(3)}=\frac{2 L^{2} m^{2}}{G_{0} r^{10}}\left(r^{4}+9 r^{2} a^{2}+9 a^{4}\right),
\end{aligned}
$$

together with the gravitational energy flux given by

$$
T^{(0)(3)}=\frac{9 L^{2} m^{2} a \Lambda\left(r^{2}+2 a^{2}\right)}{G_{0} r^{10}} .
$$

The observer encounters the singularity at $r=0$, where the curvature components as well as the components of the gravitational stress-energy tensor all diverge.

The above stress-energy tensors for the pure Kerr geometry reduce to the spherically symmetric Schwarzschild case for $a=0$; then, there is no energy flux for the radial motion of the observer and the gravitational stress-energy tensor is diagonal with elements that are proportional to $\mathrm{m}^{2} / \mathrm{r}^{6}$, as would be expected in analogy with electrodynamics. Moreover, the radial pressure is negative while the other two pressures are equal and positive in agreement with simple physical considerations based on the fact that the gravitational tidal accelerations are repulsive (i.e. they tend to pull test particles apart) along the radial direction and attractive (i.e. they tend to push test particles together) along the other two orthogonal directions in this case.

\section{Discussion}

An interesting result of this paper is the theoretical elucidation of the existence of a steady flux of gravitational energy around a rotating mass. It follows from equations (27) and (28) that

$$
T^{(0)(i)} / T^{(0)(0)} \sim 3 \frac{J}{M|x|}(\hat{J} \times \hat{\boldsymbol{x}})^{i}
$$

for large $|\boldsymbol{x}|$. This means that the current of gravitational energy has speed $v_{g}=3 a \sin \theta / r$ far from the source, as can also be seen from the results of the last section. In particular, for equatorial geodesics the ratio of equations (60) and (56) behaves as $3 a / r$ when $r \gg a$. On the other hand, the other results of the previous section, e.g. the diagonal form of equation (39), are not in conflict with this conclusion since a detailed examination of the natural tetrad system (30)-(33) of the Kerr spacetime indicates that the observer moves in the $\phi$-direction with speed $a / r$ far from the source, i.e.

$$
\frac{\mathrm{d} \phi}{\mathrm{d} \tau}=\frac{a}{(\Sigma \Delta)^{1 / 2}}
$$




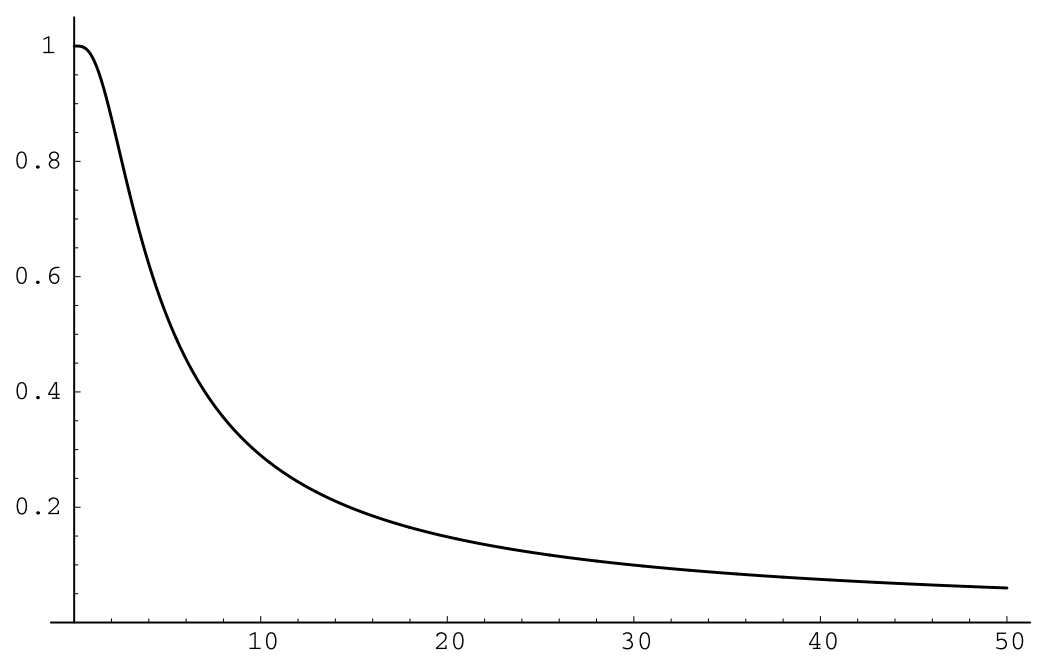

Figure 1. Plot of $v_{g}$ versus $r / a$ as given by equation (63), which represents the speed of the steady gravitational energy current in the equatorial plane of the Kerr system as perceived by observers falling freely from rest at spatial infinity. It is important to note that $v_{g} \leqslant 1$ just as in electrodynamics.

is the initial angular speed of the observer. Moreover, the flow velocity $v_{g} \hat{\phi}$ must vanish along the axis of symmetry and this is consistent with the diagonal gravitational stress-energy tensor (48) for the test observer moving along the $z$-axis.

In electrodynamics, the speed of the Poynting current is always less than or equal to the speed of light in vacuum and the analogous result holds for the GEM tensor by equation (16). Therefore, it is interesting to investigate here how $v_{g}$ behaves as a function of $r$ in the equatorial plane of the Kerr black hole as measured by the free 'radially' infalling test observers. As figure 1 demonstrates,

$$
v_{g}=\frac{3 a\left(r^{2}+a^{2}\right)^{1 / 2}\left(r^{2}+2 a^{2}\right)}{r^{4}+6 r^{2} a^{2}+6 a^{4}}
$$

increases monotonically with decreasing $r$ and reaches the speed of light at the $r=0$ singularity.

Finally, it is interesting to note that for a charged rotating mass-such as a Kerr-Newman system - there exists both an electromagnetic Poynting flux and a gravitational flux of energy moving in essentially the same way about the rotating mass. For instance, for the Kerr-Newman system we have that far from the source $v_{e m} \sim 2 a \sin \theta / r$ as $r \rightarrow \infty$. The investigation of the physical consequences of the existence of gravitational energy currents around rotating masses is beyond the scope of this work.

\section{Appendix A. Bel-Robinson tensor}

The gravitational superenergy tensor was first introduced independently by Bel and Robinson in close formal analogy with Maxwell's stress-energy tensor $[1,3,13]$. In this regard, an important consideration was the invariance of the Maxwell stress-energy tensor under duality rotations; therefore, a gravitational superenergy tensor was conceived that would be similarly invariant under duality rotations involving the gravitoelectric and gravitomagnetic fields. Some 
aspects of the early work in this direction can be found in [14]. Further work on the BelRobinson tensor is contained in [15-18], while other approaches to the stress-energy tensor for gravitation are discussed in [19]. The Bel-Robinson tensor is also related to certain quasilocal definitions of mass in curved spacetime (see [20] and the references cited therein).

Originally, spacetimes were considered with only a cosmological constant and in this case the tensor (12) already appears in the work of Bel [3]. Robinson proved that only in the Ricci-flat case would this tensor be totally symmetric and traceless. Thus later work has been restricted to $R_{\mu \nu}=0$ and the Bel-Robinson tensor is thus defined by

$$
T_{\mu \nu \rho \sigma}=\frac{1}{2}\left(R_{\mu \xi \rho \zeta} R_{v \sigma}^{\xi \zeta}+R_{\mu \xi \sigma \zeta} R_{v \rho}^{\xi \zeta}\right)-\frac{1}{16} g_{\mu \nu} g_{\rho \sigma} K
$$

That is, $\tilde{T}_{\mu \nu \rho \sigma} \rightarrow T_{\mu \nu \rho \sigma}$ since

$$
R_{\alpha \beta \rho \gamma} R_{\sigma}^{\alpha \beta \gamma}=\frac{1}{4} g_{\rho \sigma} K
$$

for $R_{\mu \nu}=0$. Here $K$ is the Kretschmann scalar, i.e. $K=R_{\mu \nu \rho \sigma} R^{\mu \nu \rho \sigma}$. In a recent paper [21], however, the Bel-Robinson tensor and the corresponding GEM Poynting vector-constructed via the Weyl part of the spacetime curvature-have been studied in a general context for arbitrary curved spacetimes.

\section{Appendix B. Landau-Lifshitz pseudotensor in Riemann normal coordinates}

The energy of a gravitational field — if it can be defined at all — is nonlocal according to general relativity. On the other hand, the Bel-Robinson tensor is locally defined. A connection could perhaps be established between these concepts if the energy-momentum pseudotensor of the gravitational field is expressed in Riemann normal coordinates about a typical event in spacetime (cf [22]).

Let $x^{\mu}$ be the Riemann normal coordinates in the neighbourhood of some point ('origin') in spacetime; then,

$$
\begin{aligned}
& g_{\mu \nu}=\eta_{\mu \nu}-\frac{1}{3} R_{\mu \alpha \nu \beta} x^{\alpha} x^{\beta}+\cdots \\
& \Gamma_{\nu \rho}^{\mu}=-\frac{1}{3}\left(R_{\nu \rho \sigma}^{\mu}+R_{\rho \nu \sigma}^{\mu}\right) x^{\sigma}+\cdots
\end{aligned}
$$

The Landau-Lifshitz pseudotensor is quadratic in the connection coefficients by construction; therefore, $t_{\mu \nu}^{L-L}$ is - at the lowest order-quadratic in Riemann normal coordinates. Hence,

$$
t_{\mu \nu, \alpha \beta}^{L-L}=\frac{c^{4}}{144 \pi G_{0}} \Theta_{\mu \nu \alpha \beta}+\cdots
$$

where $\Theta_{\mu \nu \alpha \beta}$ is symmetric in its first and second pairs of indices by construction and is given by

$$
\begin{gathered}
\Theta_{\mu \nu \alpha \beta}=\frac{1}{2}\left(R_{\mu \alpha}^{\rho \sigma} R_{\nu \sigma \rho \beta}+R_{\mu \beta}^{\rho \sigma} R_{\nu \sigma \rho \alpha}\right)+\frac{7}{2}\left(R_{\mu \rho \sigma \alpha} R_{\nu \beta}^{\rho \sigma}+R_{\mu \rho \sigma \beta} R_{\nu \alpha}^{\rho \sigma}\right) \\
-\frac{3}{8} \eta_{\mu \nu} \eta_{\alpha \beta} R_{\rho \sigma \kappa \delta} R^{\rho \sigma \kappa \delta} .
\end{gathered}
$$

This expression should be compared and contrasted with equation (A1) that expresses the Bel-Robinson tensor in a similar form. There is no simple relationship between $\Theta_{\mu \nu \alpha \beta}$ and $T_{\mu \nu \alpha \beta}$; however, one can show that

$$
\Theta_{\mu \nu \alpha \beta}-7 T_{\mu \nu \alpha \beta}=\frac{1}{16} \eta_{\alpha \beta} \eta_{\mu \nu} K+\frac{1}{4}\left(R_{\mu \alpha}^{\rho \sigma} R_{\rho \sigma \nu \beta}+R_{\mu \beta}^{\rho \sigma} R_{\rho \sigma \nu \alpha}\right)
$$

in Riemann normal coordinates. 


\section{References}

[1] Mashhoon B, McClune J C and Quevedo H 1997 Phys. Lett. A 23147

[2] Wheeler J A 1977 Phys. Rev. D 163384

Ibáñez J and Verdaguer E 1985 Phys. Rev. D 31251

Isenberg J, Jackson M and Moncrief V 1990 J. Math. Phys. 31517

Bretón N, Feinstein A and Ibáñez J 1993 Gen. Rel. Grav. 25267

[3] Bel L 1958 C. R. Acad. Sci., Paris 2471094

Bel L 1959 C. R. Acad. Sci., Paris 2481297

Bel L 1962 Cah. Phys. 1659

Debever R 1958 Bull. Soc. Math. Belg. 10112

Bel L 1962 Colloques Internationaux du CNRS (Paris) pp 119-26

[4] Matte A 1953 Can. J. Math. 51

[5] Mashhoon B 1978 Astrophys. J. 223285

[6] Mashhoon B and McClune J C 1993 Mon. Not. R. Astron. Soc. 262881

[7] Bonnor W B 1991 Phys. Lett. A 15823

[8] Mashhoon B and Quevedo H 1995 Nuovo Cimento B 110291

[9] Quevedo H 1992 Gen. Rel. Grav. 24693

[10] Mashhoon B 1992 Phys. Lett. A 1637

[11] Beem J K, Ehrlich P E and Easley K L 1996 Global Lorentzian Geometry 2nd edn (New York: Dekker) ch 2

[12] Marck J-A 1983 Proc. R. Soc. A 385431

[13] Zakharov V D 1973 Gravitational Waves in Einstein's Theory (New York: Halsted) (translated by R N Sen from the 1972 Russian edition)

[14] Rindler W and Trautman A 1987 Gravitation and Geometry ed W Rindler and A Trautman (Naples: Bibliopolis) pp 9-20

[15] Deser S 1987 Gravitation and Geometry ed W Rindler and A Trautman (Naples: Bibliopolis) pp 115-29

[16] York J W Jr 1987 Gravitation and Geometry ed W Rindler and A Trautman (Naples: Bibliopolis) pp 497-505

[17] Robinson I 1997 Class. Quantum Grav. 14 A331

[18] Bonilla M Á G and Senovilla J M M 1997 Gen. Rel. Grav. 2991

[19] Bak D, Cangemi D and Jackiw R 1994 Phys. Rev. D 495173

[20] Horowitz G T and Schmidt B G 1982 Proc. R. Soc. A 381215 Dougan A J 1992 Class. Quantum Grav. 92461

[21] Maartens R and Bassett B A 1998 Class. Quantum Grav. 15705

[22] Pirani F A E 1957 Phys. Rev. 1051089 